\title{
The Healing Effects of Autologous Mucosal Grafts in Experimentally Injured Rabbit Maxillary Sinuses
}

\author{
Murat Topdag ${ }^{1} \cdot$ Ahmet Kara $^{1} \cdot$ Esma Konuk $^{2} \cdot$ Necdet Demir $^{2} \cdot$ Murat Ozturk $^{1} \cdot$ Sebla Calıskan ${ }^{1} \cdot$ Deniz Ozlem Topdag $^{1}$ \\ Arif Ulubil $^{3} \cdot$ Ibrahim Gurkan Keskin ${ }^{1} \cdot$ Mete Iseri $^{1}$ \\ ${ }^{1}$ Department of Otorhinolaryngology, Kocaeli University Faculty of Medicine, Kocaeli; ${ }^{2}$ Department of Histology and Embryology, Akdeniz \\ University Faculty of Medicine, Antalya; ${ }^{3}$ Department of Otorhinolaryngology, Acıbadem University Faculty of Medicine, Istanbul, Turkey
}

Objectives. Healing processes of the nose and paranasal sinuses are quite complex, and poorly understood. In this study, we aimed to compare the effect of mucosal autologous grafts on the degenerated rabbit maxillary sinus mucosa with spontaneous wound healing. It is hypothesized that mucosal grafts will enhance ciliogenesis and improve the morphology of regenerated cilia.

Methods. Ten female New Zealand rabbits were included in the study. They underwent external maxillary sinus surgery through a transcutaneous approach. A total of 20 maxillary sinuses were randomly divided into 2 groups: 'spontaneous healing group' and 'autologous graft group.' The animals were sacrificed at the 14th day after the surgery. Scanning electron microscope (SEM), and light microscope were used for the evaluation.

Results. Cellular composition of the graft group is better than the spontaneous healing group. The graft group had larger areas covered with ciliary epithelium than the spontaneous healing group, and the mean length of the cilias were also longer. Additionally, there were wider cilia with abnormal morphology areas in the spontaneous healing group.

Conclusion. In our opinion, covering of the denuded areas with a graft improves re-epithelization, and may prevent the early complications after sinus surgeries.

Keywords. Nasal Mucosa; Maxillary Sinus; Rabbits

\section{INTRODUCTION}

Functional endoscopic sinus surgery, in which the surgeon can reach the paranasal sinuses directly under endoscopic vision, is a surgical method that is used quite often in the ENT (ear, nose, and throat) practice. It is used basically for the treatment of chronic sinusitis, nasal polyposis, and many other sinonasal diseases. With this surgical technique and the help of the endo-

- Received December 12, 2014

Revised February 18, 2015

Accepted February 23, 2015

- Corresponding author: Ahmet Kara

Department of Otorhinolaryngology, Kocaeli University Medicine Faculty, 41380 Umuttepe, Kocaeli, Turkey

Tel: +90-262-303-8222, Fax: +90-262-303-7003

E-mail: doktor.kbb@hotmail.com scopes, all paranasal sinuses can be accessed, and their relevant ostia can be enlarged. Despite the use of minimally invasive surgical techniques, it is not possible to prevent mucosal damage, and these mucosal damages may cause serious problems especially for the frontal recess such as synechia, osteitis, fibrosis, etc. [1-3]. Less mucosal damage or covering the skeletonized bone with a material or a graft may improve the re-epitelization period, and they may prevent complications and reoperations.

The healing process of the nose and paranasal sinuses is quite complex and poorly understood. Growth factors, fibroblasts, leukocytes, and pluripotent basal cells are known to have functions in the process of wound healing [4]. However, the respiratory epithelium having functional mucociliary structure complicates this healing process. Although there are some studies in the literature about the wound healing process of the respiratory

Copyright (c) 2016 by Korean Society of Otorhinolaryngology-Head and Neck Surgery.

This is an open-access article distributed under the terms of the Creative Commons Attribution Non-Commercial License (http://creativecommons.org/licenses/by-nc/4.0)

which permits unrestricted non-commercial use, distribution, and reproduction in any medium, provided the original work is properly cited. 
mucosa, this process is still an enigma for the surgeons who are performing the nasal and paranasal surgeries. A literature review on this topic showed that the rabbit maxillary sinus seems most appropriate for experimental studies $[5,6]$.

In this experimental study: we aimed to compare the effect of mucosal autologous grafts on the degenerated rabbit maxillary sinus mucosa with spontaneous wound healing. Scanning electron microscope (SEM), and light microscope were used for the evaluation. With these methods, this paper will be the first study that investigates the effect of mucosal grafts on the healing process of respiratory mucosa by comparing them with spontaneous healing process on the same animal.

\section{MATERIALS AND METHODS}

The research protocol was submitted and approved by the Ko- caeli University Ethics Committee for Animal Experiments. Ten female New Zealand rabbits weighing between 2.5 to $3.5 \mathrm{~kg}$ included in the study. They underwent external maxillary sinus surgery through a transcutaneous approach. A total of 20 maxillary sinuses were randomly divided into 2 groups. In 'spontaneous healing group,' the sinuses were opened surgically, a $1-\mathrm{cm}^{2}$ wide mucosa was removed, and the area kept uncovered for spontaneous healing $(n=10)$. In 'autologous graft group,' the sinuses were opened surgically, a 1- $\mathrm{cm}^{2}$ wide mucosa was removed, after hemostasis, the autologous graft which were obtained from the other side of the same animal was laid onto the degenerated mucosa, and the sinuses were closed $(n=10)$.

\section{Surgical procedure}

The rabbits were anesthetized with intraperitoneal xylazine hydrochloride $(5 \mathrm{mg} / \mathrm{kg}$ ) and ketamine hydrochloride $(35 \mathrm{mg} / \mathrm{kg}$ ). The surgeries were performed under aseptic conditions. Nasal
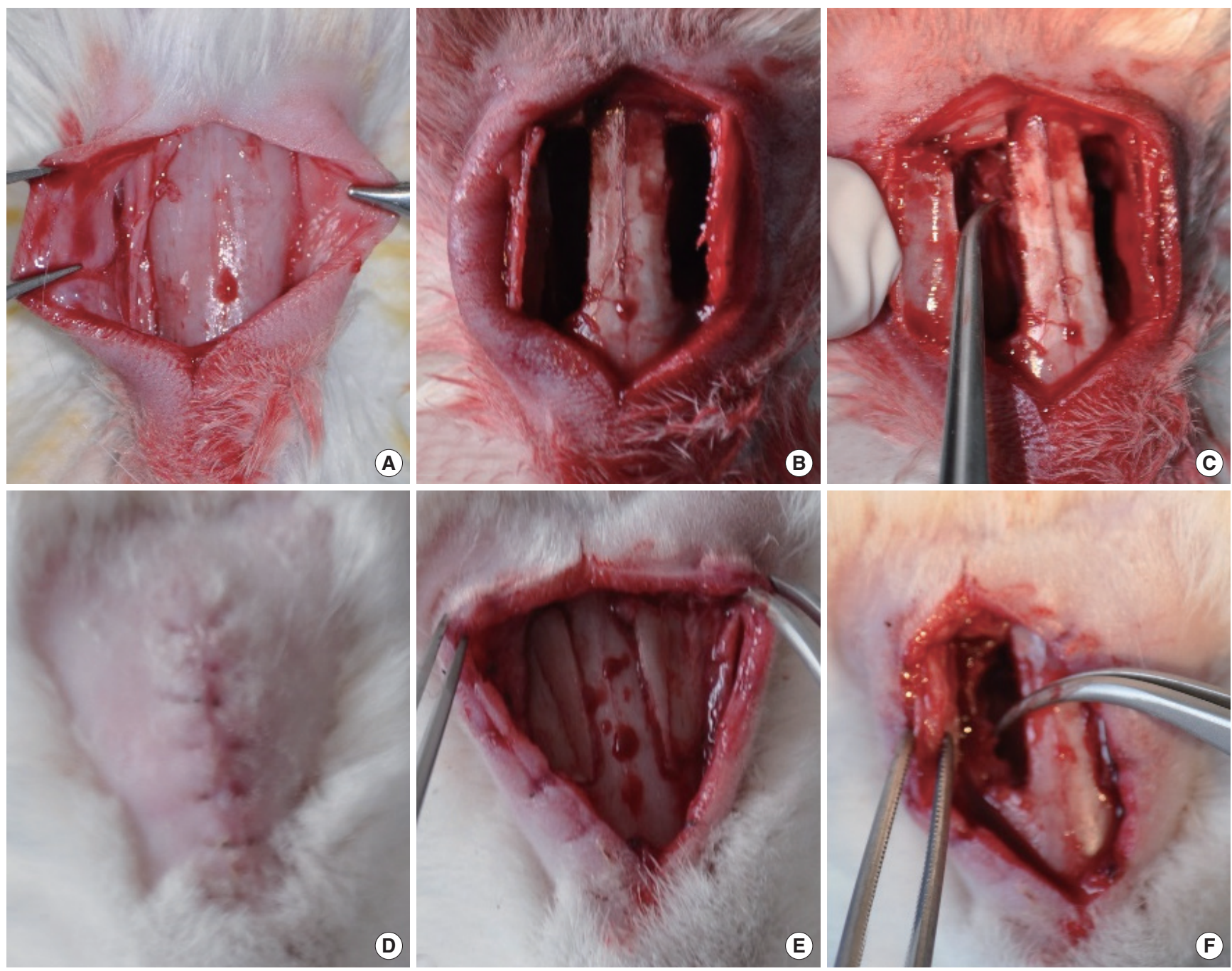

Fig. 1. Anterior walls of the maxillary sinuses (A), bilaterally bone flaps (B), elevation of an approximately $1-\mathrm{cm}^{2}$ mucosal area on the medial maxillary wall (C), healed animal (D), healed anterior wall of the sinus (E), inflamed and hypertrophic mucosa with neo osteogenesis (F). 
dorsums of the rabbits were cleaned with iodine. The procedure was started with a midline nasal dorsum incision, and the skin flaps were elevated laterally. After that, a ' $T$ ' shaped incision was made in the periosteum, and reached to the anterior walls of the maxillary sinuses (Fig. 1A). Approximately, $25 \times 8$-mm wide bone flaps were created bilaterally (Fig. 1B). The macroscopic aspect of the maxillary sinus mucosa was observed with the aid of a loop headband magnifier. An approximately $1-\mathrm{cm}^{2}$ circular mucosal area was elevated and excised on the medial maxillary wall with microsurgical equipments. Thus, an uncovered area was created on the bone, and on this side, the area kept uncovered. Using the same standardized wound model provided the standardization of the wound healing and analyzing of the histology. The excised mucosal graft was kept in sterile saline solution (Fig. 1C). After hemostasis with a piece of cotton, we passed to the other side. The same procedure was carried out in the other maxillary sinus. On this side, the uncovered area on the bone was covered with the mucosal graft that was previously harvested from the other side. The mucosa surrounding the natural ostium of the sinus was left intact to maintain the patency of sinus outflow. After that, the periosteum and the skin were sutured, and the wound was closed. The subjects were administered intraperitoneal enrofloxacin $(0.1 \mathrm{~mL} / \mathrm{kg})$ for 3 days.

All of the animals healed uneventfully (Fig. 1D), and they were sacrificed on the 14th day after the surgery with intracardiac phenobarbital $(85 \mathrm{mg} / \mathrm{kg})$. The midline incision was opened again (Fig. 1E) and the bone flaps were lateralized. Maxillary sinuses were flushed with sterile saline to clear away remaining blood. The line where we entered into the maxillary sinus was inflamed and hypertrophic with neo osteogenesis (Fig. 1F). We removed the mucosa of medial wall of the maxillary sinuses. The samples were spreaded on corks with fine needles and then fixed in $4 \%$ glutaraldehyde solution which was prepared in $0.1 \mathrm{M}$ phosphate buffer. The samples on the corks were postfixed in $1 \%$ OsO4 so- lution, dehydrated through serial submersions in grading ethyl alcohol (30\%, 50\%, 70\%, 80\%, 90\%, absolute ethyl alcohol), and then dried in a Polaron E3000 critical point drying apparatus (Quorum Technologies Ltd., Kent, UK). The dried samples were coated with gold palladium in a Polaron (SC7620) coating unit (Sputter coater), and examined in scanning electron microscopy (Zeiss LEO 1430VP, Zeiss, Oberkochen, Germany).

Each of the unlabeled samples were examined with SEM and rated on a 1 (low) to 10 (high) scale on two parameters: ciliary density (coverage of mucosal surface with cilia) and ciliary morphology (ciliary directionality, uniformity of ultrastructure). Additionally, ciliary length was measured, too. The results were compared with Mann-Whitney $U$-test by using the SPSS ver. 15.0 (SPSS Inc., Chicago, IL, USA). A $P$-value lower than 0.05 was considered significant.

\section{RESULTS}

To our observations, we can say that the mucosa and the bone tissue on the anteromedial walls of spontaneous healing group were more inflamed and hypertrophic with neo osteogenesis. Additionally, the mucosal adhesions in the spontaneous healing group were more than in the graft group.

The light microscopic examinations showed that the graft group contained more and taller epithelial cells than spontaneous healing group. Basal cells constituted a continuous cell line over the lamina propria in both of the groups. Cellular composition of the graft group was better, and cellular diversification did not occur sufficiently in the spontaneous healing group (Fig. 2).

Examinations with SEM showed that nasal ciliary epithelium developed in both of the groups, and ciliary improvement did not differ too much between the groups. There were both areas covered with ciliary epithelium and connective tissue areas with-
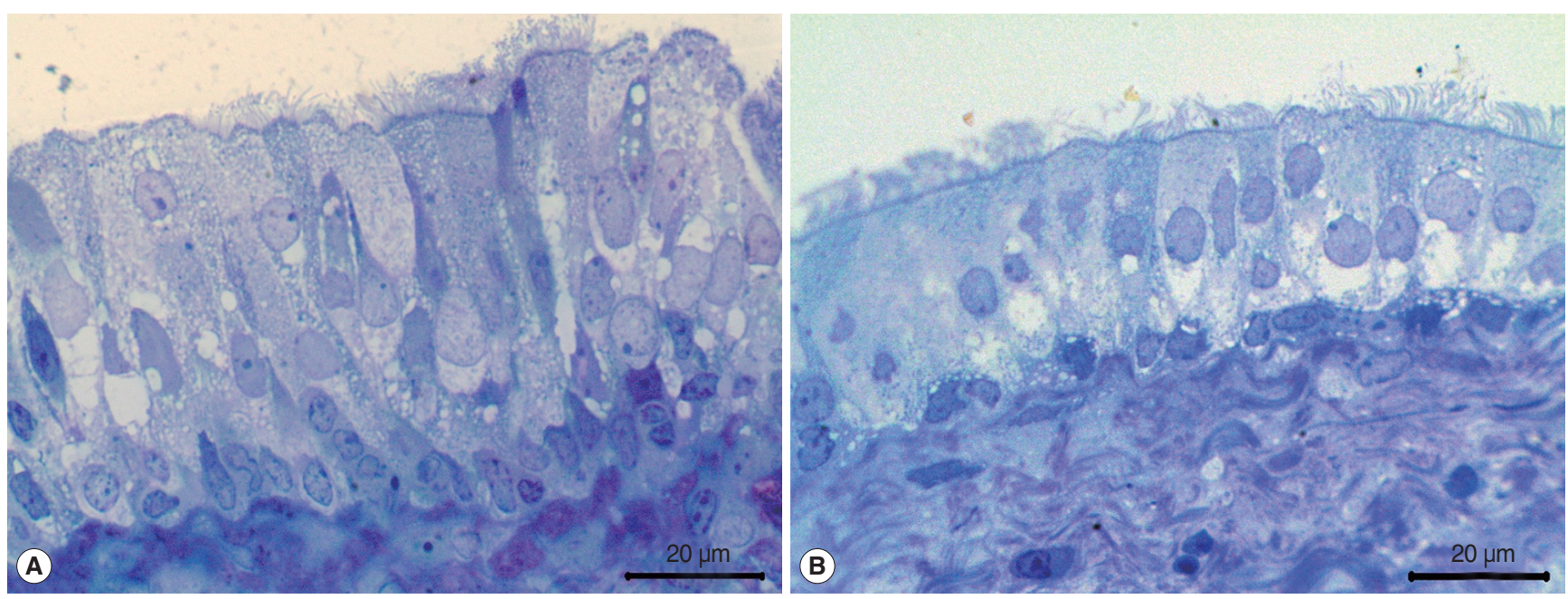

Fig. 2. Light microscopic examinations (toluidin blue) showed graft group (A) contained more and higher epithelial cells than spontaneous healing group $(\mathrm{B})$. 
out epithelium in each groups. Because the samples were folded during preparation, we could not standardize the results quantitatively. However, based on our observations, we can state that the graft group had larger areas covered with ciliary epithelium than the spontaneous healing group. Ten measurements were performed per specimens and the length of the cilia is statistically longer in the graft group $(P<0.0001)$ when compared with Mann-Whitney $U$-test. This finding suggested that the cilia in the graft group were more mature and sophisticated than the spontaneous healing group (Table 1). The mean thickness of the epithelium in the graft group was $30.2 \mu \mathrm{m}$ (standard deviation, 4.35) and $68.3 \mu \mathrm{m}$ (standard deviation, 6.1) in the spontaneous healing group. The difference was statistically significant $(P<0.0001)$ which is compared with Mann-Whitney $U$-test. This result may explain the hypertrophic appearance of the mucosa in the secondary healing group. Apical surface buds that show the early stages of epithelial ciliary developments were found in both of the groups in the ciliary epithelium (Fig. 3). The cell cordons in the graft group fully spreaded over the surface to form a superficial cover. In the spontaneous healing group, epithelial dispersion was in different areas, and unable to create a cover (Fig. 4). If the groups were compared in terms of ciliary morphology, we determined both cilias with normal morphology and thinner, curled, and filamentary cilias in each of the groups (Fig. 5). However, the spontaneous healing group showed wider areas of cilia with abnormal morphology.

Table 1. Length of the cilias in both of the groups

\begin{tabular}{llc}
\hline Length of the cilias & $\begin{array}{l}\text { Autologous } \\
\text { graft group }\end{array}$ & $\begin{array}{c}\text { Spontaneous } \\
\text { healing group }\end{array}$ \\
\hline Mean $\pm S D(\mu)$ & $5.07 \pm 0.89$ & $3.85 \pm 0.39$ \\
Median $($ range $)(\mu)$ & $5.10(3.47-7.45)$ & $3.70(3.08-4.48)$ \\
\hline
\end{tabular}

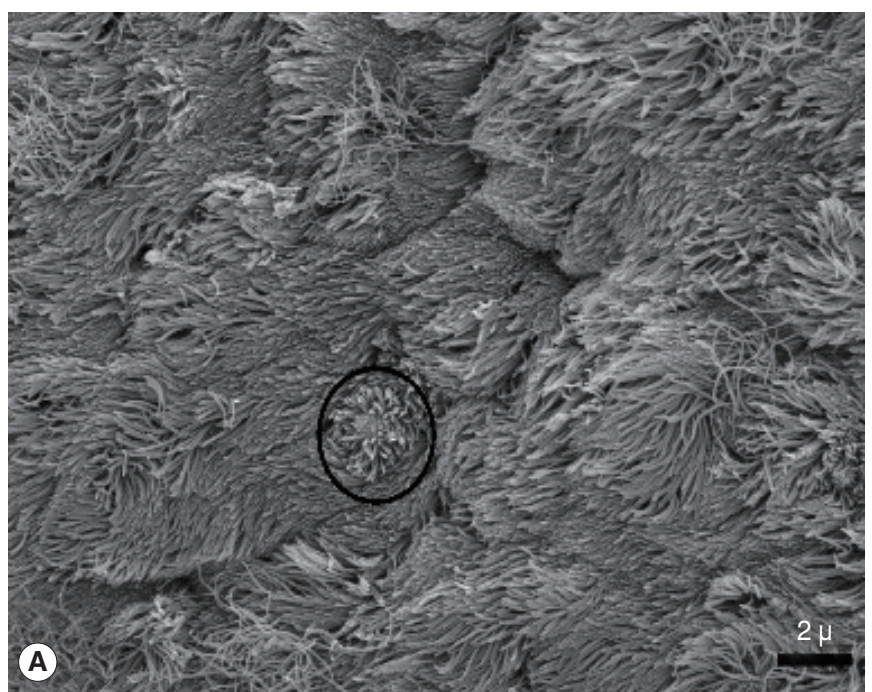

\section{DISCUSSION}

Although the current surgical approaches to the paranasal sinuses are less invasive and controlled than the externally approaches, the intact mucosa is still included in the resection material with affected tissues. Today, we are still waiting for spontaneous healing period after endoscopic surgeries. The new epithelium usually cannot be fully regenerated to obtain functional mucociliary activity [7-9]. The mentioned spontaneous healing period may be the reason for the permanent nasal blockages, and nasal dryness after these kinds of surgeries, especially in patients who underwent extensive sinus surgeries.

Regenerated mucosa after sinus surgery is the subject of curiosity for the surgeons who are interested in this surgery. Using the rabbit maxillary sinuses to investigate the paranasal sinus pathologies is mostly accepted by the investigators [5,6]. Exposure of the rabbit maxillary sinuses is described several times in different publications $[10,11]$. Our study was designed based on these described techniques.

Forsgren et al. [12] studied the regeneration of rabbit maxillary sinus after experimental operative removal of the mucosa. Although the denuded areas have re-epithelialized, regeneration of the subepithelial glands was not complete and the glands located in normal mucosa were replaced by dense connective tissue. In Benninger's study, 15 rabbits underwent unilateral removal of maxillary sinus mucosa to evaluate sinus mucosal regeneration and cilia motility. Histopathological findings showed; fibrosis, decreased sero-mucinous glands, and significant inflammation. In the electron microscopic examinations, complex and edematous cilia and fewer than normal cilia per unit area were determined [13]. Peter performed two consecutive studies that investigated the effect of topical retinoic acid on the mucosal

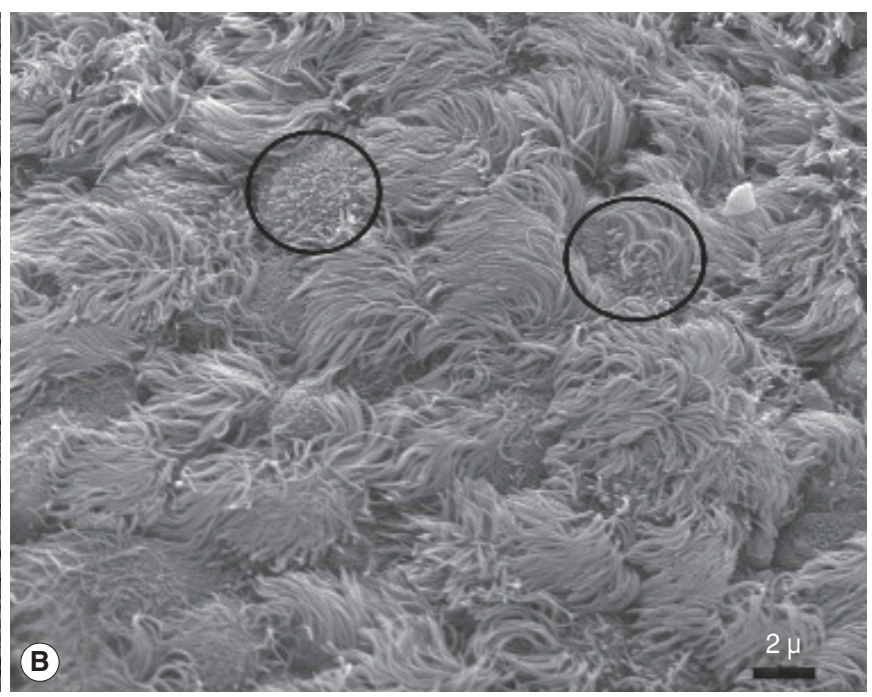

Fig. 3. Scanning electron microscope images of epithelial ciliary organizations. Graft (A) and spontaneous healing group (B). Apical surface buds are seen early of the epitelial ciliary development both of the graft and spontaneous healing groups. They are showed with ring in the A and $\mathrm{B}$ panels. 

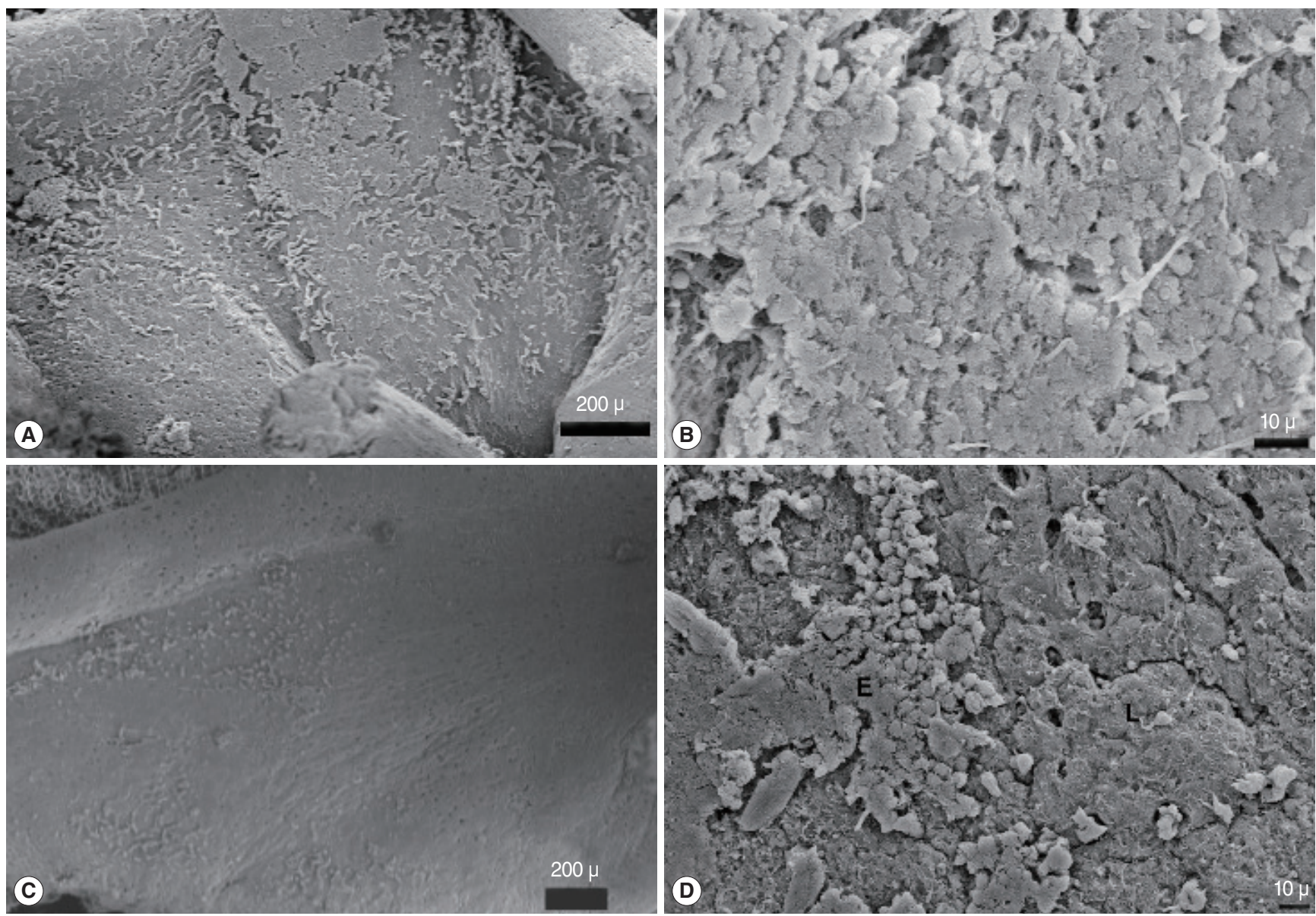

Fig. 4. Cell cordons in the graft group were spreaded over the surface to form a superficial cover; graft (A, B), epithelial dispersion was in different areas and unable to create a cover; spontaneous healing group (C, D).
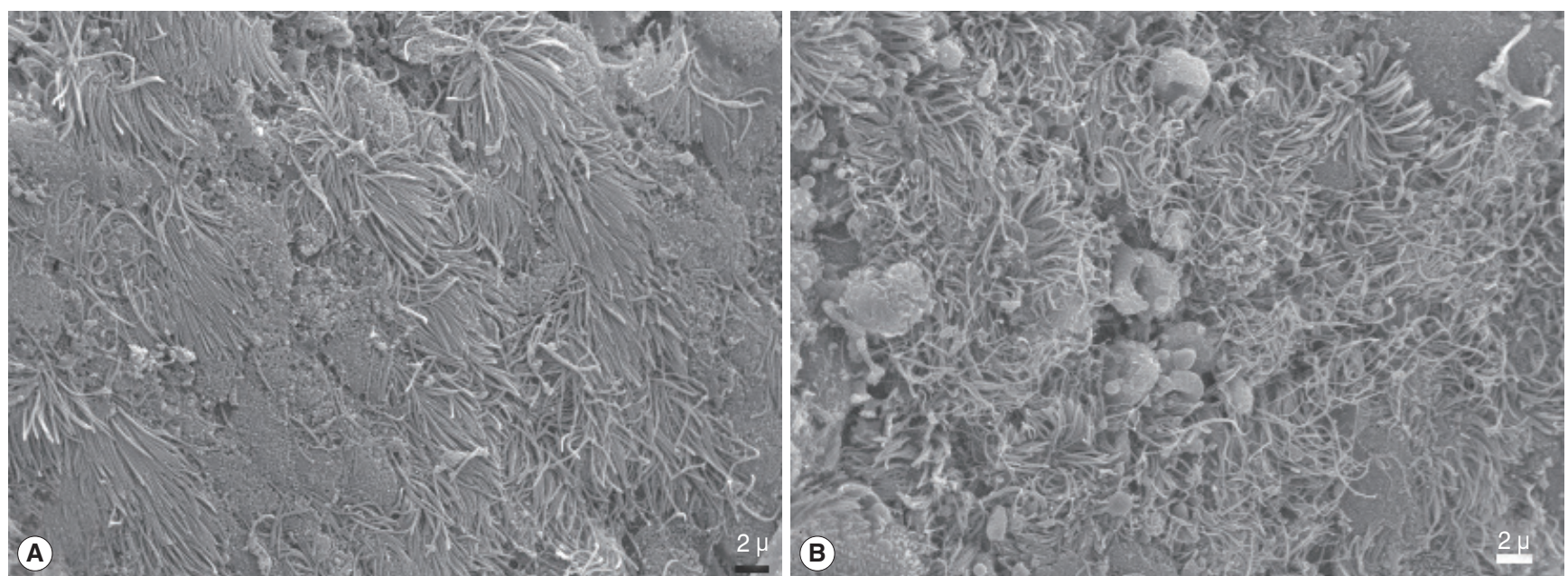

Fig. 5. Abnormal ciliary formations in the scanning electron microscope images. Graft (A) and spontaneous healing group (B).

wound healing in a rabbit model, and found that retinoic acid enhances ciliogenesis $[14,15]$. Inanl studied on 30 patients $(24$ patients with chronic sinusitis and 6 controls) to determine his- tological findings in the maxillary sinus mucosa, and ciliary activity within the nasal cavity by measuring the speed of mucociliary transport after endoscopic sinus surgery. Findings showed 
the mucosa have not improved completely 10 weeks after surgery [7]. Beule et al. $[16,17]$ performed two studies that investigated the effect of dexamethasone on mucosal wound healing and osteoneogenesis after a healing period of 23 days after surgical trauma and found benefical effects of the therapy. Hosemann et al. [18] studied the effects of autografts for frontal cerebrospinal fluid leaks and found that eight weeks after the operation the outline of the transplant could no longer be exactly identified by microscopy and the graft had been completely transformed into scar tissue with intercalated bony trabeculae. We think that the reason of this fibrosis may be due to their technique such as; transferring of a 5 -mm graft on a 2-mm defect and administration of the fibrin glue onto the graft. Another study with free mucosal grafts was performed by Hildenbrand et al. [19] at 2012 with 16 patients undergoing Draf III drainage. In that study, the investigators modified the Lothrop procedure by covering the bare bone with mucosal transplants from the nasal septum. At the end of the follow-up period (minimum follow-up, 12 months; mean, 25.6 months), 94\% of frontal sinus neo-ostia remained open. As a result of this study, the investigators concluded that, the modified technique reduce the granulations and crusting, reduce the need of intensive and frequent cleaning of the nose, and increase the patients' comfort.

In our study, rabbit maxillary sinus mucosa was damaged bilaterally and investigations were performed 2 weeks after the surgery. Using the same animal to investigate both spontaneous healing and the effect of graft on healing period provided us to exclude the respective factors of different animals. Follow-up period was determined as 2 weeks because we aimed to investigate the postoperative early complications such as: fibrosis, osteitis, adhesions, etc. The samples were folded during preparations so that we could not standardize some of the results quantitatively. Excision of the mucosa with bone could have been appropriate to prevent mucosal folding, and this could have provided quantitative assessment. We think that this knowledge may be important for future studies. Actually, this is an anatomical study, so measuring of the physiologic factors including mucociliary flow, humidification, temperature flux, airflow could not be possible in our experimental design and this deficiency limited to determine the physiological effects of the autologous grafts. Further researches on this topic may be beneficial.

Some of the paranasal sinus surgeries such as; Draf procedures, endoscopic medial maxillectomy, skull base surgeries causes large bony areas without respiratory mucosa. We can comment that covering the bony areas with an own mucosal graft may reduce the granulations, osteoneogenesis, synechias, and restenosis of the sinus ostiums. It was determined that mucosal graft group had better histological findings than spontaneous healing group in the current study. We believe that the progenitor cells in the basal layer of the mucosa are the reason for the better healing. In addition, grafting provide a normal thickness mucosa except hypertrophic and inflamed mucosal healing. Normally healed mu- cosa may reduce some of the postoperative problems such as nasal dryness or chronic nasal blockage.

Although it is a bit difficult to find and transfer the autologous mucosal grafts in clinical practice, previous studies showed the availability of this technique [19]. We think that it is very important to show that a free mucosal graft can live and transform to a functional respiratory mucosa on peeled bone which was not shown in previous studies with histological examinations. The data obtained from this study can be used for further studies. For example, this information may be combined with stem cell studies that are very intriguing for lots of investigators nowadays. A matter of fact, there is already an ongoing study about this issue in our clinic.

In conclusion, with this experimental study, we aimed to get some information about the mucosal healing period and the effect of the grafts on this process. Although there are lots of unknown factors about this issue, in our opinion: covering of the denuded bone tissue with a graft improves re-epithelization, and may prevent from the early complications after sinus surgeries.

\section{CONFLICT OF INTEREST}

No potential conflict of interest relevant to this article was reported.

\section{ACKNOWLEDGMENTS}

This article has been published as a poster at the 10th National Rhinology Congress, in Antalya, Turkey, 2014.

\section{REFERENCES}

1. Thawley SE, Deddens AE. Transfrontal endoscopic management of frontal recess disease. Am J Rhinol. 1995 Nov-Dec;9(6):307-11.

2. Wormald PJ.The axillary flap approach to the frontal recess. Laryngoscope. 2002 Mar;112(3):494-9.

3. Wormald PJ, Chan SZ. Surgical techniques for the removal of frontal recess cells obstructing the frontal ostium. Am J Rhinol. 2003 JulAug;17(4):221-6.

4. Watelet JB, Bachert C, Gevaert P, Van Cauwenberge P. Wound healing of the nasal and paranasal mucosa: a review. Am J Rhinol. 2002 Mar-Apr;16(2):77-84.

5. Casteleyn C, Cornillie P, Hermens A, Van Loo D, Van Hoorebeke L, van den BroeckW, et al.Topography of the rabbit paranasal sinuses as a prerequisite to model human sinusitis. Rhinology. 2010 Sep;48(3): $300-4$.

6. Koybasioglu A, Ileri F, Beder L, Inal E. Anatomy of the rabbit maxillary sinus. Kulak Burun Bogaz Bas Boyun Cerrahi Derg. 1997;5:41-4.

7. Inanli S, Tutkun A, Batman C, Okar I, Uneri C, Sehitoglu MA. The effect of endoscopic sinus surgery on mucociliary activity and healing of maxillary sinus mucosa. Rhinology. 2000 Sep;38(3):120-3.

8. Bassiouny A, Atef AM, Raouf MA, Nasr SM, Nasr M, Ayad EE. Ultrastructural ciliary changes of maxillary sinus mucosa following 
functional endoscopic sinus surgery: an image analysis quantitative study. J Laryngol Otol. 2003 Apr;117(4):273-9.

9. Guo Y, Majima Y, Hattori M, Seki S, Sakakura Y. Effects of functional endoscopic sinus surgery on maxillary sinus mucosa. Arch Otolaryngol Head Neck Surg. 1997 Oct;123(10):1097-100.

10. Perez AC, Cunha Junior Ada S, Fialho SL, Silva LM, Dorgam JV, Murashima Ade A, et al. Assessing the maxillary sinus mucosa of rabbits in the presence of biodegradable implants. Braz J Otorhinolaryngol. 2012 Dec;78(6):40-6.

11. Swibel Rosenthal LH, Benninger MS, Stone CH, Zacharek MA. Wound healing in the rabbit paranasal sinuses after Coblation: evaluation for use in endoscopic sinus surgery. Am J Rhinol Allergy. 2009 May-Jun;23(3):360-3.

12. Forsgren K, Stierna P, Kumlien J, Carlsoo B. Regeneration of maxillary sinus mucosa following surgical removal. Experimental study in rabbits. Ann Otol Rhinol Laryngol. 1993 Jun;102(6):459-66.

13. Benninger MS, Schmidt JL, Crissman JD, Gottlieb C. Mucociliary function following sinus mucosal regeneration. Otolaryngol Head Neck Surg. 1991 Nov;105(5):641-8.
14. Maccabee MS, Trune DR, Hwang PH. Paranasal sinus mucosal regeneration: the effect of topical retinoic acid. Am J Rhinol. 2003 May-Jun;17(3):133-7.

15. Hwang PH, Chan JM. Retinoic acid improves ciliogenesis after surgery of the maxillary sinus in rabbits. Laryngoscope. 2006 Jul;116(7): 1080-5.

16. Beule AG, Scharf C, Biebler KE, Gopferich A, Steinmeier E, Wolf E, et al. Effects of topically applied dexamethasone on mucosal wound healing using a drug-releasing stent. Laryngoscope. 2008 Nov;118(11): 2073-7.

17. Beule AG, Steinmeier E, Kaftan H, Biebler KE, Gopferich A, Wolf E, et al. Effects of a dexamethasone-releasing stent on osteoneogenesis in a rabbit model. Am J Rhinol Allergy. 2009 Jul-Aug;23(4):433-6.

18. Hosemann W, Goede U, Sauer M. Wound healing of mucosal autografts for frontal cerebrospinal fluid leaks: clinical and experimental investigations. Rhinology. 1999 Sep;37(3):108-12.

19. Hildenbrand T, Wormald PJ, Weber RK. Endoscopic frontal sinus drainage Draf type III with mucosal transplants. Am J Rhinol Allergy. 2012 Mar-Apr;26(2):148-51. 